\section{Percursos e obstáculos na Rede de Atenção à Saúde: trajetórias assistenciais de mulheres em região de saúde do Nordeste brasileiro}

\author{
Healthcare trajectories and obstacles faced by \\ women in a health region in Northeast Brazil
}

\author{
Itinerarios y obstáculos en la Red de Atención de \\ Salud: trayectorias asistenciales de mujeres en \\ la región de salud del Nordeste brasileño
}

Jôse Ribas Galvão 1 Patty Fidelis de Almeida 2 Adriano Maia dos Santos 1 Aylene Bousquat 3

\section{Resumo}

O artigo tem como objetivo avaliar a organização e o acesso à Rede de Atenção à Saúde em uma região de saúde, na perspectiva das usuárias. Foram construídas trajetórias assistenciais de mulheres com lesão intraepitelial escamosa de alto grau do colo do útero, adscritas a diferentes modalidades de atenção primária à saúde (APS) de zonas urbana e rural, em municípios do interior e da sede de região de saúde do Nordeste do Brasil. As mulheres utilizavam a APS como serviço de busca regular para ações preventivas e assistenciais, mas reportaram barreiras de acesso para consultas médicas, sobretudo nas zonas rurais. Avaliações positivas foram vinculadas ao acolhimento e à representação da unidade básica de saúde/unidade de saúde da família (UBS/ USF) como lócus disponível para cuidados. Percepção de baixa resolutividade da APS esteve ligada à demora para o agendamento das referências, abastecimento irregular/insuficiente de medicamentos e rotatividade de médicos. As mulheres indicaram dificuldade de acesso à atenção especializada, mais evidente nos municípios do interior da região, com utilização de serviços públicos e privados. Todas as usuárias da sede da região realizaram exames de confirmação diagnóstica e cirurgia pelo Sistema Único de Saúde. Apoio de familiares, amigos e politicos atravessaram as trajetórias assistenciais. Comunicação interprofissional foi quase inexistente e entre profissional/usuária, precária. A rede regionalizada apresentou-se desarticulada e com fluxos desordenados, não garantindo acesso oportuno às usuárias dos municípios do interior e apresentando dificuldades adicionais àquelas da área rural, mesmo no município sede, desvelando a incompletude dos arranjos regionais e a manutenção de desigualdades de acesso inter e intramunicipal.

Assistência Integral à Saúde; Integração de Sistemas; Regionalização; Neoplasias do Colo do Útero

\author{
Correspondence \\ P. F. Almeida \\ Departamento de Planejamento em Saúde, Instituto de Saúde \\ Coletiva, Universidade Federal Fluminense. \\ Rua Marquês do Paraná 303, 3o andar, Niterói, RJ \\ 24030-900, Brasil. \\ pattyfidelis@id.uff.br \\ ${ }^{1}$ Instituto Multidisciplinar em Saúde, Universidade Federal da \\ Bahia, Vitória da Conquista, Brasil. \\ 2 Instituto de Saúde Coletiva, Universidade Federal Fluminense, \\ Niterói, Brasil. \\ 3 Faculdade de Saúde Pública, Universidade de São Paulo, São \\ Paulo, Brasil.
}




\section{Introdução}

A organização dos serviços de saúde em rede tem sido considerada condição importante à garantia do direito à saúde em diversos sistemas. No caso do Sistema Único de Saúde (SUS) é essencial para a consecução dos princípios da universalidade, equidade e integralidade. Nenhum ponto de atenção isolado, qualquer que seja o nível de complexidade, é suficiente para resolver as demandas de saúde de um indivíduo ou população, que depende da constituição de redes integradas de serviços, redes regionalizadas ou serviços integrados para a garantia dos cuidados 1,2 .

As redes de atenção regionalizadas deveriam garantir respostas às necessidades de saúde dos usuários, uma vez que podem ser consideradas a essência da organização e do funcionamento do sistema de saúde. Para tanto, devem suprimir barreiras aos itinerários regionais ao facilitar o acesso, com oferta suficiente de serviços e racionalidade nos gastos ${ }^{3}$. Para que isso aconteça, são imprescindíveis investimentos em todos os níveis de atenção, desde o fortalecimento da atenção primária à saúde (APS) à qualificação da atenção especializada, bem como a incorporação de uma reforma hospitalar capaz de integrar e reorientar as redes e seus diversos modelos de provisão, segundo as necessidades sociais em contextos regionais 2 .

No Brasil, diversos estudos têm avaliado as iniciativas de constituição de redes regionalizadas 4,5,6. Requer-se, cada vez mais, nos cenários nacional e internacional, uma visão integrada dos níveis de atenção visto que o cuidado envolve ações de diferentes serviços e profissionais. Só uma resposta articulada e coordenada é capaz de garantir um bom resultado assistencial final para o indivíduo e a comunidade 2,7. Avaliar a capacidade de articulação e integração das Redes de Atenção à Saúde (RAS) é, sem dúvida, um grande desafio para pesquisadores e formuladores de políticas e, em certa medida, central para compreender o atual estágio de implementação do SUS.

O presente artigo propõe-se a avaliar a organização e o acesso à RAS, na perspectiva das usuárias, por meio da construção de trajetórias assistenciais de mulheres em municípios do interior e da sede de região de saúde do Nordeste do país.

\section{Método}

Trata-se de estudo avaliativo, com abordagem qualitativa, por meio da construção das trajetórias assistenciais de usuárias diagnosticadas com lesão intraepitelial escamosa de alto grau do colo uterino (HSIL), considerada precursora do câncer do colo de útero (CCU) e cujo cuidado requer uma efetiva integração dos serviços de saúde. Avaliações sobre o cuidado integrado às usuárias com lesões percursoras do CCU têm indicado insuficiências em diversos países da América Latina 8,9. Vale recordar que a detecção e o tratamento das lesões precursoras causadoras do CCU, quarta causa de mortes por câncer em mulheres no Brasil, sobretudo em regiões de menor desenvolvimento socioeconômico 10, demandam intervenções que promovam a integralidade do cuidado, articulando detecção precoce e garantia de acesso a procedimentos diagnósticos e terapêuticos em tempo oportuno e com qualidade em toda a RAS 11.

As trajetórias assistenciais têm gerado evidências para a avaliação da articulação dos diversos serviços do sistema de saúde 12 , dos processos de trabalho, da organização e funcionamento da rede e da qualidade do cuidado 5,13, circunscrevendo-se ao percurso trilhado nas redes assistenciais formais 14 . Tal abordagem não apresenta o mesmo objetivo e escopo dos itinerários terapêuticos, que envolvem o conjunto de planos e ações utilizados pelos usuários para lidar com a enfermidade para além dos limites dos serviços de saúde, com base nos três sistemas de cuidados propostos por Kleinman 15: profissional, popular e informal.

As trajetórias assistenciais foram construídas por meio de entrevistas em profundidade com 26 mulheres diagnosticadas com HSIL em 2016, entrevistadas em 2017, com vistas a diminuir o viés de memória e assegurar tempo razoável para a confirmação diagnóstica e tratamento. As entrevistas foram complementadas com consulta aos prontuários na APS e análise de resultados de exames e procedimentos.

O estudo foi realizado em uma região de saúde de um estado do Nordeste do país, composta por 19 municípios, com população de 632.708 habitantes, sendo 32,5\% em zona rural 16. O município sede 
da região tem uma ampla zona rural, com 284 povoados e 12 distritos. Para avaliar a organização e o acesso à RAS com base no evento traçador (HSIL), foram selecionados três municípios (identificados por letras) com características distintas na região de saúde (Quadro 1): município A, sede, maior porte populacional e disponibilidade de serviços especializados e hospitalares; município B, que fica a 1 hora e 20 minutos da sede e é dependente do município A para exames de confirmação diagnóstica e tratamento das mulheres com HSIL; e município C, localizado a 30 quilômetros da sede e dependente dos serviços para a realização do anatomopatológico do colo uterino e tratamento. A inclusão de municípios com distintas inserções na região é central para identificar e analisar os diferentes obstáculos enfrentados regionalmente, do interior para a sede, de áreas rurais para urbanas.

\section{Quadro 1}

Caracterização dos municípios selecionados - Região de Saúde, Nordeste, Brasil, 2018.

\begin{tabular}{|c|c|c|c|c|}
\hline \multirow[t]{2}{*}{ Município } & \multicolumn{3}{|c|}{ Características gerais } & \multirow[t]{2}{*}{ Características da rede assistencial } \\
\hline & População & $\begin{array}{c}\text { Cobertura ESF } \\
(\%)\end{array}$ & IDH & \\
\hline A & 306.866 & 43,53 & 0,678 & $\begin{array}{c}\text { Sede da região de saúde; } \\
\text { Concentra praticamente toda a oferta de atenção especializada e } \\
\text { hospitalar da região de saúde; } \\
\text { Central de marcação de consultas e regulação, com médico regulador; } \\
\text { Laboratório de saúde pública - realiza a análise das lâminas do citopatológico; } \\
\text { Policlínica municipal - referência para ginecologia oncológica, confirmação diagnóstica } \\
\text { e tratamento cirúrgico ambulatorial. Referência para os demais municípios; } \\
\text { Hospital municipal - referência para confirmação diagnóstica e cirurgia; } \\
\text { Hospital conveniado ao SUS que realiza cirurgias para tratamento do CCU. } \\
\text { Referência para os demais municípios; } \\
\text { Laboratório privado - estabelecimento conveniado ao } \\
\text { Sue realiza exame anatomopatológico; } \\
\text { quimidade de alta complexidade em oncologia - tratamento do CCU - cirurgia, } \\
\text { em unidade hospitalar estadual e em unidade hospitalar privada conveniada. }\end{array}$ \\
\hline $\mathrm{B}$ & 44.701 & 100,00 & 0,604 & $\begin{array}{l}\text { Central de marcação de consultas; } \\
\text { Leitura das lâminas do citopatológico realizada em laboratório privado conveniado } \\
\text { ao SUS, localizado em Salvador; } \\
\text { Policlínica municipal de referência, com oferta de consultas em ginecologia, para } \\
\text { mulheres que apresentem resultados alterados - não realiza colposcopia nem biópsia; } \\
\text { Colposcopia e biópsia pactuadas no município sede, por sua vez o anatomopatológico } \\
\text { é realizado em serviço privado contratado no próprio município; } \\
\text { Referência para tratamento do CCU no município sede e em Salvador, num } \\
\text { Centro/Clínica de especialidade em oncologia. }\end{array}$ \\
\hline C & 34.788 & 100,00 & 0,551 & $\begin{array}{l}\text { Central de marcação de consultas; } \\
\text { Laboratório citopatológico próprio; } \\
\text { Ambulatório de especialidades municipal de referência, com oferta } \\
\text { de colposcopia e biópsia; } \\
\text { Anatomopatológico e tratamento das mulheres são pactuados com a } \\
\text { sede da região de saúde. }\end{array}$ \\
\hline
\end{tabular}

CCU: câncer de colo uterino; ESF: Estratégia Saúde da Família; IDH: índice de desenvolvimento humano; SUS: Sistema Único de Saúde. Fonte: Bahia (2018), entrevista exploratória com as coordenações das Secretarias de Saúde dos municípios estudados. 
As mulheres diagnosticadas com HSIL, em 2016, foram identificadas no Sistema de Informação do Câncer (SISCAN) nos municípios B e C e, no município A, no livro do Laboratório Municipal de Citopatologia, uma vez que na sede da região de saúde não havia sido implantado o SISCAN. No município A, identificou-se a população-alvo de 86 usuárias; 11 no C e 4 no município B. Posteriormente, foi verificada a distribuição das mulheres conforme o tipo de unidade/modalidade de APS e o contexto territorial (urbano ou rural) (Tabela 1). A seleção das mulheres ocorreu no interior de cada modalidade de APS, por meio de sorteio, exceto no município B e na zona rural do município C, onde todas foram entrevistadas. Em seguida, as usuárias foram contatadas e convidadas a participar da entrevista com o apoio das equipes de APS. O número de entrevistadas foi definido por saturação de conteúdo, contemplando-se as distintas inserções no território regional. Características socioeconômicas das participantes do estudo, algumas das quais importantes para a compreensão das trajetórias assistenciais, são apresentadas na Tabela 1.

As entrevistas, realizadas no domicílio, com duração de aproximadamente 1 hora, foram gravadas, transcritas e posteriormente analisadas por meio de dimensões para a avaliação de aspectos da organização e do acesso à rede regionalizada, com destaque para a APS na função de coordenação do cuidado, selecionadas baseando-se em estudos sobre o tema 4,5,17,18 e por categorias emergentes da experiência das mulheres, quais sejam: porta de entrada preferencial pela APS, abrangência e resolubilidade da APS, plano de cuidado, integração vertical e acesso aos serviços de referência e comunicação interprofissional. O tratamento do material empírico foi orientado pela análise de conteúdo 19.

O estudo foi aprovado pelo Comitê de Ética em Pesquisa da Universidade Federal Fluminense (Parecer 2.207.909). As usuárias foram identificadas por números, modalidade de APS (unidade de saúde da família - USF ou unidade básica de saúde - UBS), localidade de residência (zona urbana - ZU ou rural - ZR) e por município de origem (A, B e C). Nomes de pessoas e serviços foram suprimidos.

\section{Resultados}

Os resultados estão apresentados por meio das experiências das usuárias em diferentes níveis da RAS (APS, serviços especializados, hospitalares, sistemas de apoio e logístico), sendo as dimensões para análise da organização e acesso às RAS e coordenação do cuidado pela APS tratadas no interior das trajetórias assistenciais. Para tanto, o Quadro 2 apresenta a síntese dos principais resultados por dimensões de análise e "falas expressivas" que imprimem densidade à experiência de uso dos serviços.

\section{Início da trajetória: da APS à atenção especializada}

Em relação à dimensão de porta de entrada preferencial pela APS, as usuárias referiram usar as UBS como serviço de busca regular para as ações preventivas e assistenciais. Identificaram importante dificuldade de acesso às consultas médicas, cuja marcação, tanto em unidades da zona urbana dos três municípios, quanto na rural do município $C$, exigia enfrentamento de filas, sem garantia de vaga. Situação mais grave na zona rural da sede da região, cujas equipes estavam presentes nos povoados uma vez ao mês. A marcação de consultas, em geral, era intermediada pelo agente comunitário de saúde (ACS). Por vezes, era necessário o deslocamento de mais de uma hora da zona rural até uma UBS tradicional no centro do município sede. As usuárias indicaram problemas em relação à "abertura" da porta de entrada via APS ao apontar situações que poderiam ser acolhidas e resolvidas pelas equipes, cuja negativa resultava em peregrinação entre hospitais e Unidades de Pronto Atendimento (UPA).

A baixa resolutividade e abrangência da APS foi mais referida por mulheres da zona urbana e esteve mais presente no discurso das usuárias do município C. Nos três municípios, esse problema foi atrelado às dificuldades de agendamento de consultas e exames especializados, ao abastecimento irregular/insuficiente de medicamentos e à rotatividade de médicos. Apesar de muitas falas apontarem fragilidades na APS, esta foi avaliada positivamente pela maioria das mulheres em função do acolhimento, diálogo dos profissionais e à representação da UBS/USF como lócus disponível para a busca de cuidados.

Em todos os casos, as equipes de APS desenvolveram ações de controle do CCU, ora mais abrangentes, ora mais restritas. Metade das mulheres referiu não ter participado de atividade educativa 
Tabela 1

Caracterização das usuárias entrevistadas por município e modalidade da atenção primária à saúde (APS). Região de Saúde, Nordeste, Brasil, 2018.

\begin{tabular}{|c|c|c|}
\hline Município/Modalidade de APS & Usuárias identificadas & Usuárias entrevistadas \\
\hline \multicolumn{3}{|l|}{ A } \\
\hline UBS zona rural & 2 & 2 \\
\hline UBS zona urbana & 19 & 4 \\
\hline USF zona rural & 16 & 5 \\
\hline USF zona urbana & 49 & 4 \\
\hline \multicolumn{3}{|l|}{ B } \\
\hline USF zona rural & 3 & 3 \\
\hline USF zona urbana & 8 & 4 \\
\hline \multicolumn{3}{|l|}{ C } \\
\hline USF zona urbana & 4 & 4 \\
\hline Total de usuárias & 101 & 26 \\
\hline Características das mulheres entrevistadas & $\mathbf{n}$ & $\%$ \\
\hline \multicolumn{3}{|l|}{ Idade (anos) } \\
\hline$<25$ & 1 & 3,8 \\
\hline $25-44$ & 15 & 57,8 \\
\hline $45-64$ & 9 & 34,6 \\
\hline 65 & 1 & 3,8 \\
\hline \multicolumn{3}{|l|}{ Cor autorreferida } \\
\hline Branca & 7 & 26,9 \\
\hline Parda & 12 & 46,2 \\
\hline Preta & 7 & 26,9 \\
\hline \multicolumn{3}{|l|}{ Nível educacional } \\
\hline Analfabetismo & 10 & 38,5 \\
\hline Ensino Fundamental incompleto & 11 & 42,3 \\
\hline Ensino Fundamental completo & 1 & 3,8 \\
\hline Ensino Médio incompleto & 3 & 11,6 \\
\hline Ensino Médio completo & 1 & 3,8 \\
\hline \multicolumn{3}{|l|}{ Ocupação remunerada } \\
\hline Sim & 9 & 34,6 \\
\hline Não & 17 & 65,4 \\
\hline \multicolumn{3}{|l|}{ Rendimento mensal do lar (salário mínimo) } \\
\hline Até 1 & 16 & 61,5 \\
\hline Até 2 & 6 & 23,1 \\
\hline Até 3 & 3 & 11,6 \\
\hline Não informou & 1 & 3,8 \\
\hline \multicolumn{3}{|l|}{ Benefício social } \\
\hline Sim & 22 & 84,6 \\
\hline Não & 4 & 15,4 \\
\hline
\end{tabular}

Fonte: elaboração própria.

sobre o tema e as demais, esporadicamente. Na maior parte das trajetórias assistenciais (21) não houve convocação para a realização do exame citopatológico do colo uterino. Esse exame foi realizado por iniciativa da mulher ou por incentivo de familiares, muitas vezes diante da manifestação de sintomas. A UBS, porta de entrada para acesso ao citopatológico, apresentou barreiras para pouco mais da metade (14) das mulheres, sobretudo para aquelas que viviam na área rural da sede e urbana do município C. 
Quadro 2

Matriz e resultados da análise da organização e acesso às Redes de Atenção à Saúde e coordenação do cuidado pela atenção primária à saúde (APS) em região de saúde na perspectiva das usuárias, 2018.

\begin{tabular}{|c|c|c|}
\hline Dim & Síntese dos principais resultados & Falas expressivas \\
\hline \multirow{5}{*}{$\begin{array}{l}\text { Porta de entrada } \\
\text { preferencial pela } \\
\text { APS }\end{array}$} & $\begin{array}{l}\text { Serviço de busca regular para ações } \\
\text { preventivas e assistenciais }\end{array}$ & $\begin{array}{l}\text { "Eu passo no posto assim (...) eu passo com o médico, às vezes, ele passa alguma pomada } \\
\text { pra mim passar, eu passo. Passa algum comprimido, eu uso também (...). Assim, agora com } \\
\text { a (enfermeira) eu vou, ela quer que eu vou fazer um outro preventivo (...). Já fiz pré-natal" } \\
\text { (E4-USF-ZR-B). } \\
\text { "(...) porque até agora ainda não fumei nenhum cigarro, que eu fumava 15, 20. Até agora } \\
\text { não tenho vontade de fumar. E foi pelo posto. Eu comecei o tratamento terça-feira (...), aí, tive } \\
\text { que por o adesivo" (E3-USF-ZU-B). }\end{array}$ \\
\hline & $\begin{array}{l}\text { Dificuldade de acesso às consultas } \\
\text { médicas }\end{array}$ & $\begin{array}{l}\text { "Bom, acho assim, que pela demora que eles (a equipe) demora pra vim (no povoado), } \\
\text { eles tinha que vim pra ficar o dia todo e atender todo mundo assim, conforme cada um tá } \\
\text { precisando, né? (...) a maioria das vezes, eles marca que vem e não vem. E quando vem não } \\
\text { atende todo mundo" (E16-USF-ZR-A). }\end{array}$ \\
\hline & $\begin{array}{l}\text { Marcação de consultas intermediada } \\
\text { pelo ACS }\end{array}$ & $\begin{array}{l}\text { "Assim, eu chego, aí o (ACS) vai na minha casa e avisa tem médico tal dia. Aí eu chego aqui } \\
\text { com o cartão da família e falo '(ACS), eu quero passar com a enfermeira'. Aí se tiver vaga ele } \\
\text { coloca meu nome lá" (E16-USF-ZR-A). }\end{array}$ \\
\hline & Longos trajetos à unidade $d$ & $\begin{array}{l}\text { "Pra eu vir praqui (UBS) eu pego ônibus. Eu saí de lá (povoado onde reside) faltava dez pra } \\
\text { seis. Cheguei aqui faltava, mais ou menos, dez pra sete. Uma hora" (E10-UBS-ZR-A). }\end{array}$ \\
\hline & $\begin{array}{l}\text { Dificuldade de acesso ao exame } \\
\text { citopatológico do colo do útero }\end{array}$ & $\begin{array}{l}\text { "A gente demorava pra fazer um preventivo, era aquela burocracia (...) tinha que surgir vaga } \\
\text { e não sei o que, era bastante tempo pra marcar pra gente passar pra fazer" (E25-USF-ZU-C). }\end{array}$ \\
\hline \multirow{4}{*}{$\begin{array}{l}\text { Abrangência e } \\
\text { resolubilidade } \\
\text { da APS }\end{array}$} & $\begin{array}{l}\text { Atividades de educação em saúde } \\
\text { ausentes ou esporádicas }\end{array}$ & "Nunca participei não. Se já teve lá, nunca me avisaram não" (E23-USF-ZU-C). \\
\hline & $\begin{array}{c}\text { Baixa resolutividade atrelada à } \\
\text { dificuldade de agendamento para } \\
\text { atenção especializada, assistência } \\
\text { farmacêutica e rotatividade de } \\
\text { médicos, implicando gastos privados }\end{array}$ & $\begin{array}{l}\text { "Só a única reclamação que a gente tem é sobre os exames, que vai pra lá, e a demora } \\
\text { (...). Exame que demora um ano, dois anos, e que nem vem. Às vezes, a gente resolve } \\
\text { (os problemas na unidade), mas, às vezes, não, porque se for um caso de urgência, como } \\
\text { quando eu adoeci, eu precisava de cardiologista e era urgente, eu tive que partir pra onde? } \\
\text { Pra pagar" (E10-UBS-ZR-A). }\end{array}$ \\
\hline & $\begin{array}{l}\text { Satisfação com o atendimento de } \\
\text { profissionais da APS }\end{array}$ & $\begin{array}{l}\text { "Ah, com toda ruindade e tudo, eu dou nota } 10 \text { ainda. Pelo menos, elas sabe conversar com } \\
\text { você, entendeu? Só num acompanha a gente em nada, mas (...)" (E26-USF-ZU-C). }\end{array}$ \\
\hline & $\begin{array}{l}\text { Desenvolvimento de ações } \\
\text { desarticuladas e incompletas }\end{array}$ & $\begin{array}{l}\text { "Aí, a gente que se interessa por a gente mesmo né? Pega e vai. Entendeu? Elas num fala } \\
\text { nada não. Se você num procura (pela coleta), você num faz não. É por a gente mesmo. Se, no } \\
\text { caso, eu vejo que eu tô precisando, eu vou atrás" (E26-USF-ZU-C). } \\
\text { "Aí ela falou assim, só que a doutora não vai abrir não (o resultado), eu vou marcar pra você } \\
\text { ir lá na policlínica para passar com doutor (ginecologista)" (E1-USF-ZU-B). }\end{array}$ \\
\hline
\end{tabular}

(continua)

A busca ativa das usuárias com resultado alterado e a posterior avaliação por meio de consulta aconteceu na maior parte dos casos, com exceção das residentes no município B e algumas da zona rural. Em relação à integração vertical e ao acesso aos serviços de referência, todas as usuárias foram encaminhadas à atenção especializada pela APS para consulta com especialista ou diretamente para a realização da confirmação diagnóstica. Em seis trajetórias assistenciais, o agendamento foi realizado diretamente pelas usuárias na central de marcação de consultas e em uma no próprio serviço especializado. Algumas mulheres não passaram por consulta com o profissional na APS e foram encaminhadas à atenção especializada desconhecendo o motivo, o que resultou em abandono do tratamento por uma das usuárias. Apenas quatro relataram recebimento de relatório de referência da APS a ser entregue ao especialista. 
Quadro 2 (continuação)

\begin{tabular}{|c|c|c|}
\hline Dimensão & Síntese dos principais resultados & Falas expressivas \\
\hline \multirow{6}{*}{$\begin{array}{l}\text { Integração } \\
\text { vertical e acesso } \\
\text { aos serviços de } \\
\text { referência }\end{array}$} & $\begin{array}{l}\text { Agendamento do primeiro acesso à } \\
\text { atenção especializada pela APS }\end{array}$ & $\begin{array}{l}\text { "Ela (enfermeira da APS) pegou os papel (solicitação de consulta com } \\
\text { ginecologista) que ficou aqui, e falou assim que marcava e, quando marcou, levou, } \\
\text { já tava marcado" (E10-UBS-ZR-A). }\end{array}$ \\
\hline & $\begin{array}{l}\text { Agendamento de retornos, exames } \\
\text { especializados e procedimentos } \\
\text { cirúrgicos realizados pelas usuárias }\end{array}$ & $\begin{array}{l}\text { "Na central de marcação (onde marcou a colposcopia). Aí ele (ginecologista) carimbou, } \\
\text { com o carimbo dele porque é mais rápido, né. Aí eu fui (na Central de Marcação) marquei, } \\
\text { com, acho que no outro dia eu já fui fazer também" (E8-UBS-ZU-A). }\end{array}$ \\
\hline & $\begin{array}{l}\text { Difícil acesso à confirmação } \\
\text { diagnóstica e ao tratamento para as } \\
\text { mulheres dos municípios do interior }\end{array}$ & $\begin{array}{l}\text { "Aí, fui aguardando, aguardando (a marcação para anatomopatológico). Como te falei, o } \\
\text { SUS num tava saindo nada, e se ficasse velho demais também num ficava bom, aí me deram } \\
\text { conselho, né? Que não podia ficar mais de dois mês não, e que o SUS ia demorar de voltar de } \\
\text { novo. Aí, eu fui, levei, fiz pago, particular" (E20-USF-ZR-C). } \\
\text { "Eu vou Ihe falar, o que eu venho passando, cê não sabe (...) desde que eu descobri } \\
\text { que tinha esse problema, o que eu passei viajando pra tudo quanto é canto aí, já } \\
\text { passei por tanto médico e ninguém me fala nada! Tudo fala a mesma coisa, que } \\
\text { eu tenho que fazer (a cirurgia), mas cadê?! Ninguém faz! Não resolve. Não resolve } \\
\text { o problema. É difícil!" (E4-USF-ZU-B). }\end{array}$ \\
\hline & $\begin{array}{l}\text { Utilização de serviços públicos e } \\
\text { privados simultaneamente }\end{array}$ & $\begin{array}{l}\text { “(...) ele falou (ginecologista da rede pública), pelo SUS tinha, mas pelo SUS ia demorar } \\
\text { muito, entendeu?' Aí ele falou, você paga uma taxinha e aqui você vai, passa por ele (...) } \\
\text { que é mais rápido. Aí eu fui, paguei cento e cinquenta reais e aí passei por ele (cirurgião } \\
\text { que atendia na rede pública e privada)” (E5-UBS-ZU-A). }\end{array}$ \\
\hline & Não garantia de transporte sanitário & $\begin{array}{l}\text { "Na verdade, de van, porque as van anda mais rápido e marcaram pra cedo demais, outra } \\
\text { hora marcava pra à tarde pra gente não perder, pra não chegar atrasado, porque é muita } \\
\text { dificuldade pra você conseguir uma coisa e depois você perder. A gente tinha que dar um } \\
\text { jeito de guardar dinheiro pra pagar passagem pra não perder, entendeu?" (E4-USF-ZU-B). }\end{array}$ \\
\hline & $\begin{array}{c}\text { Satisfação das mulheres com os } \\
\text { serviços da atenção especializada e } \\
\text { hospitalar }\end{array}$ & $\begin{array}{l}\text { "Eu fiquei satisfeita. Dou nota (...) nota grande pra eles. Porque eles cuida da gente bem, } \\
\text { conversa bem, trata a gente bem" (E2-USF-ZU-B). } \\
\text { "Oh, menina, o atendimento lá no hospital (conveniado na sede), pra mim, eu vou te falar, } \\
\text { foi ótimo. Eu nunca tinha ido num hospital pra mim ser atendida igual eu fui atendida. } \\
\text { Excelente atendimento. De enfermeira, a médico, a tudo. Que quem fez a cirurgia foi } \\
\text { Dr. (cirurgião). Foi excelente" (E10-UBS-ZR-A). }\end{array}$ \\
\hline \multirow{3}{*}{$\begin{array}{l}\text { Plano de } \\
\text { cuidado }\end{array}$} & $\begin{array}{l}\text { Apoio da família, amigos e políticos } \\
\text { nas trajetórias assistenciais das } \\
\text { usuárias pela rede de atenção à } \\
\text { saúde }\end{array}$ & $\begin{array}{l}\text { "Só foi o preventivo aí que eu fiz pelo SUS. Os outro foi tudo particular. Mas foi minha mãe } \\
\text { que me deu o dinheiro mais minha irmã" (E1-USF-ZU-B). } \\
\text { "E aí foi até o vereador que pagou pra mim (os exames de confirmação diagnóstica). Que } \\
\text { eu não vou mentir, eu tava sem o dinheiro. Porque o que eu pego eu pagava logo a farmácia, } \\
\text { pagava um gás, uma água, luz, um mercado, quando eu comprava" (E3-USF-ZU-B). }\end{array}$ \\
\hline & Deficiente seguimento pela APS & $\begin{array}{c}\text { "E me encaminhou pro tratamento. Só foi até aí. Agora, daí pra cá também, ninguém } \\
\text { me procurou pra saber se fiz a cirurgia. Não tem acompanhamento (...) Porque, se a } \\
\text { gente foi encaminhado daqui pra fazer o tratamento, deveria ter uma pessoa pra tá } \\
\text { sabendo como que a gente tá, né? Se a gente tá precisando de mais algum } \\
\text { tratamento, alguma coisa" (E10-UBS-ZR-A). }\end{array}$ \\
\hline & $\begin{array}{l}\text { Comunicação precária entre } \\
\text { profissional e usuária }\end{array}$ & $\begin{array}{l}\text { "Porque esse exame meu, eu acho assim, porque não entendo muito dessas coisas, já eles } \\
\text { entende, né? Então quando esse exame chegou (citopatológico), eu acho que antes de marcar } \\
\text { (para atenção especializada), eles devia ter dado um jeito pra conversar comigo e me } \\
\text { explicar direitinho o que que tava acontecendo, né?" (E16-USF-ZR-A). }\end{array}$ \\
\hline \multirow{2}{*}{$\begin{array}{l}\text { Comunicação } \\
\text { interprofissional }\end{array}$} & $\begin{array}{l}\text { Quase inexistente o envio de } \\
\text { relatório da APS ao especialista }\end{array}$ & $\begin{array}{l}\text { “Não (entregou relatório de referência para especialista). Só o exame (resultado do } \\
\text { citopatológico) que ela (enfermeira da APS) mandou levar" (E14-USF-ZU-A). }\end{array}$ \\
\hline & Contrarreferência inexistente & $\begin{array}{l}\text { “Deu não (relatório da ginecologista para APS). Só vinha mesmo o resultado da biópsia. } \\
\qquad \text { Num me deu papel nenhum não” (E23-USF-ZU-C). }\end{array}$ \\
\hline
\end{tabular}

A: município A; ACS: agente comunitário de saúde; B: município B; C: município C; CCU: câncer do colo do útero; E: entrevista; UBS: unidade básica de saúde (tradicional); USF: unidade de saúde da família; ZR: zona rural; ZU: zona urbana. 
O tempo de espera para acesso à consulta com especialista variou de 2 a 60 dias. As entrevistadas da zona rural da sede da região relataram tempo de espera superior à maioria das usuárias deste município. Os agendamentos para procedimentos e consultas para atenção especializada das usuárias de zona rural, quando realizados pela equipe de APS, eram mais demorados se comparados aos efetuados pela própria usuária na central de marcação de consultas.

A partir do encaminhamento para atenção especializada, a APS desapareceu da maior parte das trajetórias, conforme Figura 1, cujos tons mais escuros representam as interdições dos percursos terapêuticos. Em relação ao plano de cuidado, as poucas usuárias que retornaram à UBS/USF o fizeram por iniciativa própria, geralmente pela necessidade de marcação de consultas e exames para atenção especializada. Não foi identificado, na maioria dos casos, profissional de referência na APS que tenha coordenado o cuidado na RAS.

Para o acesso à referência na atenção especializada e hospitalar foi verificada a utilização de serviços públicos e privados, nos três municípios, além de trajetórias em curso, interrompidas ou já concluídas (Figura 1). Nos municípios B e C, a procura pelo setor privado ocorreu por dificuldades de acesso à rede pública, por insuficiência ou demora na marcação. Ao contrário, no município sede, a busca por serviços privados, geralmente, ocorria por recomendação de profissionais da rede pública para agilizar o tratamento ou por iniciativa da própria usuária.

$\mathrm{Na}$ maior parte das trajetórias, o primeiro acesso à atenção especializada foi garantido na rede pública do município de residência, via APS. A partir de então, a marcação de procedimentos e consultas passou a ser realizada diretamente pelas mulheres na central de marcação de consultas e Secretarias Municipais de Saúde (SMS), na maioria dos casos, salvo no município C, onde retornos ao ginecologista, quando houve, foram agendados pela USF.

No município B, com menor disponibilidade de serviços, apenas uma usuária conseguiu realizar exames de confirmação diagnóstica (colposcopia e biópsia) no setor público, na sede da região, ainda assim, entre idas e vindas para agendamento na SMS. As demais usuárias do município B, após peregrinarem entre SMS e serviços de saúde, trilharam caminhos no setor privado do próprio município, com desembolso direto.

Parte das mulheres do município C relatou menor dificuldade para a realização de colposcopia e biópsia na rede pública. Entretanto, duas efetuaram desembolso direto. Outra teve a trajetória assistencial interrompida, nesse município, por não conseguir marcação e não dispor de recursos próprios, mesmo um ano e oito meses após a coleta do citopatológico. Essa usuária relatou uso de remédio caseiro para o tratamento da lesão. Para todas as usuárias residentes no município sede foram asseguradas colposcopia e biópsia no SUS (Figura 1).

Apenas para as entrevistadas do município $\mathrm{C}$ e três do A os exames de confirmação diagnóstica foram realizados na mesma consulta, por iniciativa de ginecologista que atendia nos serviços públicos. A maioria das mulheres do município sede realizou colposcopia em uma consulta e, uma vez detectada a lesão, buscou a central de marcação de consultas novamente para agendamento da biópsia, com custos pessoais e financeiros mais penosos às mulheres da zona rural.

Das 26 entrevistadas, 19 realizaram o anatomopatológico do colo uterino até o momento das entrevistas. O exame foi ofertado por laboratórios conveniados ao SUS para todas as mulheres da sede e uma do município C (Figura 1, E26-USF-ZU-C), que conseguiu a marcação por influência de político e de um funcionário público. As demais usuárias (7) pagaram pelo exame na rede privada.

Os maiores tempos de espera para a realização do anatomopatológico, no município sede da região, foram apontados por três entrevistadas da zona rural (entre 30 e 60 dias). A consulta para avaliação do resultado ocorreu na rede pública para todas as usuárias da sede, embora dúvidas em relação à marcação e agendamentos incorretos tenham contribuído para o aumento do tempo de espera.

As usuárias do interior, de posse da confirmação diagnóstica, geralmente dirigiam-se à clínica privada na sede ou no próprio município, com recursos próprios, diante da expectativa da não garantia de avaliação na rede pública. Tal fato ocorreu mesmo no município C, onde havia especialista para a realização da colposcopia e biópsia, e no B, que também dispunha de ginecologista. Na policlínica pública do município sede foram realizadas as consultas de avaliação de todas as suas mulheres e de uma do município B. A maior parte das mulheres não realizou os exames e as consultas com o mesmo ginecologista, mesmo quando utilizaram serviços públicos. 
Figura 1

Representação gráfica das trajetórias assistenciais de mulheres com lesão precursora do câncer de colo do útero na rede de atenção em região de saúde. Região de Saúde, Nordeste, Brasil, 2018.

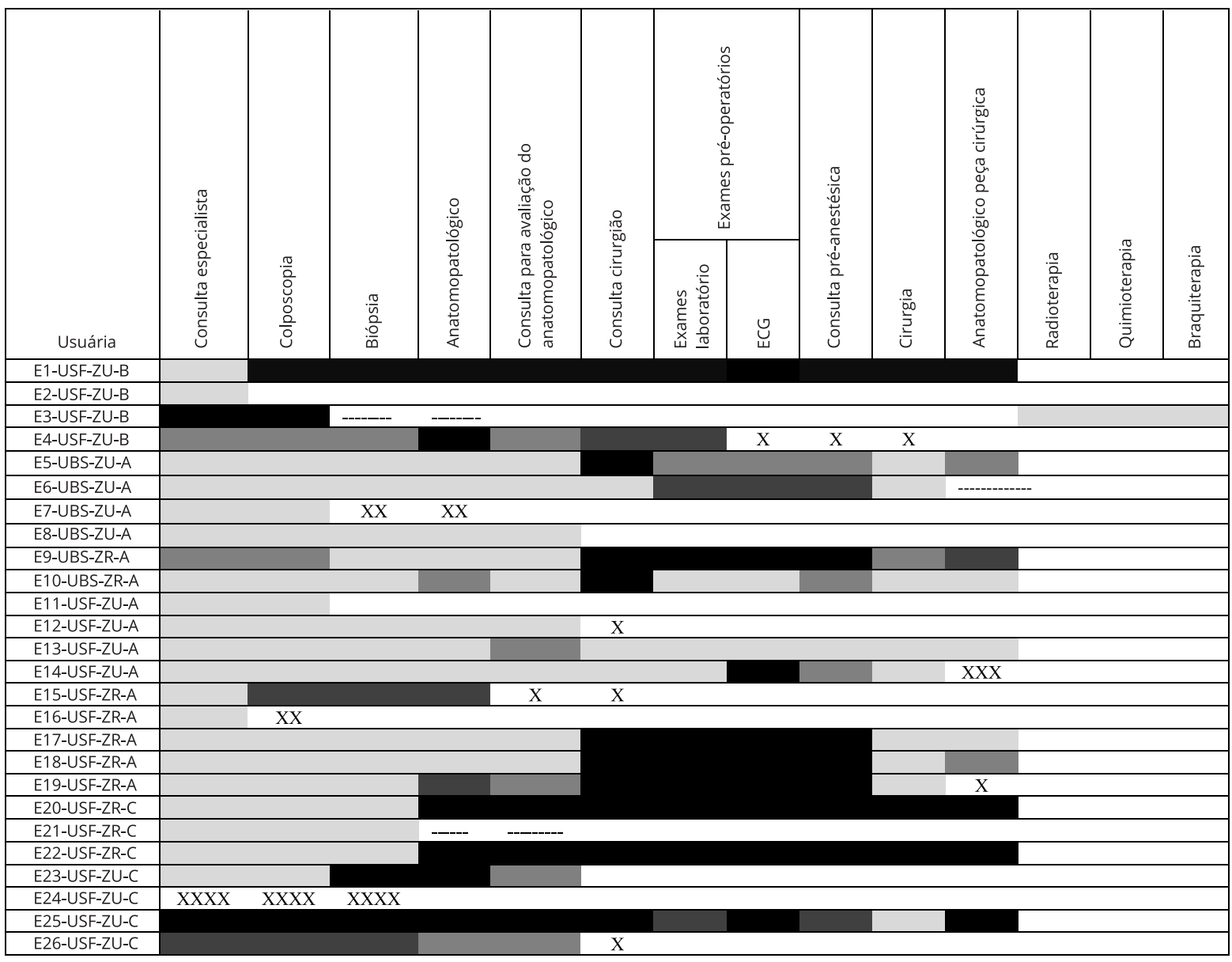

Não indicado pelo profissional

Acesso livre

Acesso lento

Barreiras de acesso

Acesso via setor privado não conveniado ao SUS
X Usuária aguardando

XXX Usuária desistiu por dificuldade

XXXX Usuária não conseguiu acesso

------- Usuária não soube informar

A: município A; B: município B; C: município C; E: entrevista; ECG: eletrocardiograma; UBS: unidade básica de saúde (tradicional); USF: unidade de saúde da família; ZR: zona rural; ZU: zona urbana.

Fonte: construção a partir das entrevistas. 


\section{Continuando o percurso, avançando à rede hospitalar: o desembolso direto como forma de garantir o acesso ao tratamento}

A maioria das mulheres, após avaliação do anatomopatológico, foi encaminhada ao cirurgião (Figura 1), mas somente duas relataram ter recebido relatório do ginecologista para ser entregue a este profissional. Apenas duas entrevistadas (entre as $18 \mathrm{com}$ exame avaliado) não tiveram solicitação para cirurgião e uma não soube responder. Uma usuária do município C, que não pôde pagar pela consulta, ainda aguardava pelo agendamento, aproximadamente um ano após o citopatológico alterado (Figura 1, E26-USF-ZU-C).

A maior parte das usuárias do município A (seis entre nove) buscou consulta com cirurgião em serviço privado por indicação de um ginecologista que as havia atendido no SUS, com o argumento de agilizar o tratamento. O cirurgião indicado para a consulta privada também atendia pelo SUS, e outras usuárias não mencionaram dificuldades de acesso pela rede pública. Uma das entrevistadas que recebeu essa recomendação agendou a consulta no setor privado e posteriormente a enfermeira da equipe de APS a encaminhou via SUS, em tempo oportuno. Todas as usuárias que passaram por consulta privada com esse cirurgião realizaram as cirurgias pelo SUS. Algumas delas interpretaram esse fato como favor do profissional.

Para as 14 entrevistadas que realizaram consultas com cirurgião houve indicação de procedimento cirúrgico (histerectomia ou conização). A maior parte das usuárias dos municípios B e C realizou exames pré-operatórios na rede privada com recursos próprios, no município de residência ou na sede da região. Entre as dez usuárias do município sede, metade teve tais exames garantidos na rede pública. As demais usuárias da sede que realizaram o pré-operatório no setor privado, em geral, o fizeram por recomendação do cirurgião, com o argumento de adiantar e viabilizar a realização da cirurgia pelo SUS.

A avaliação pré-anestésica foi ofertada em serviços públicos para a maioria das usuárias da sede e somente para uma do município C (por influência de conhecido) (Figura 1, E25-USF-ZU-C). As demais, incluindo as do município B, o de menor disponibilidade de serviços, acessaram clínicas ou hospitais na sede da região, com recursos próprios. Uma das mulheres do município A relatou que, após pagar pela consulta com anestesista, teve a avaliação negada pelo hospital que realizaria a cirurgia que, desta vez, a encaminhou para um prestador público (Figura 1, E10-UBS-ZR-A). Os retornos ao cirurgião, quando atendidas pelo SUS, foram marcados geralmente no próprio serviço hospitalar.

Todas as usuárias da sede da região realizaram a cirurgia pelo SUS, sendo a maioria no hospital conveniado, com garantia, para a maior parte, do exame anatomopatológico de peça cirúrgica, em laboratório conveniado. Houve relatos de obstáculos no acesso a esse exame por mulheres do município A, pela demora na marcação e por preenchimento incorreto da solicitação, o que resultou em idas e vindas entre laboratório, hospital, central de marcação de consultas e, em um caso (Figura 1, E14-USF-ZU-A), culminou no descarte do material. Na região, o padrão era que a própria mulher se responsabilizasse por levar a peça cirúrgica ao laboratório.

Usuárias dos municípios B e C, que necessitaram de cirurgia, as realizaram em hospitais privados da sede, com desembolso direto, por obstáculos no acesso via SUS. Apenas uma entrevistada do município C realizou em serviço conveniado, ainda assim, após prévio pagamento de consulta com cirurgião. Os anatomopatológicos da peça cirúrgica também foram feitos na sede, com recursos próprios, exceto para uma residente do município $\mathrm{C}$, que realizou no próprio município, também mediante pagamento.

As mulheres submetidas à cirurgia na rede pública tiveram acompanhamento pelo mesmo cirurgião. Houve um caso de peregrinação pela rede especializada de uma usuária da sede, que apresentou sangramento após a histerectomia, resultando em pagamento por consulta no setor privado com o mesmo especialista que a atendia na rede pública. Nenhuma entrevistada relatou recebimento do relatório de especialistas (ginecologista ou cirurgião) a ser entregue na APS.

Apenas uma usuária foi tratada com quimioterapia e radioterapia (Figura 1, E3-USF-ZU-B) e precisou pagar por medicamentos e realização de exames necessários à continuação da quimioterapia, pois a rede não garantiu resultados em tempo oportuno.

A Figura 2 apresenta, respeitadas as singularidades dos casos, as trajetórias assistenciais mais comuns de mulheres nos municípios A e C. Devido à diversidade dos caminhos percorridos pelas 


\section{Figura 2}

Fluxograma das trajetórias assistenciais na Rede de Atenção à Saúde, municípios A e C. Região de Saúde, Nordeste, Brasil, 2018.

2a) Usuária do município A - sede da região de saúde.

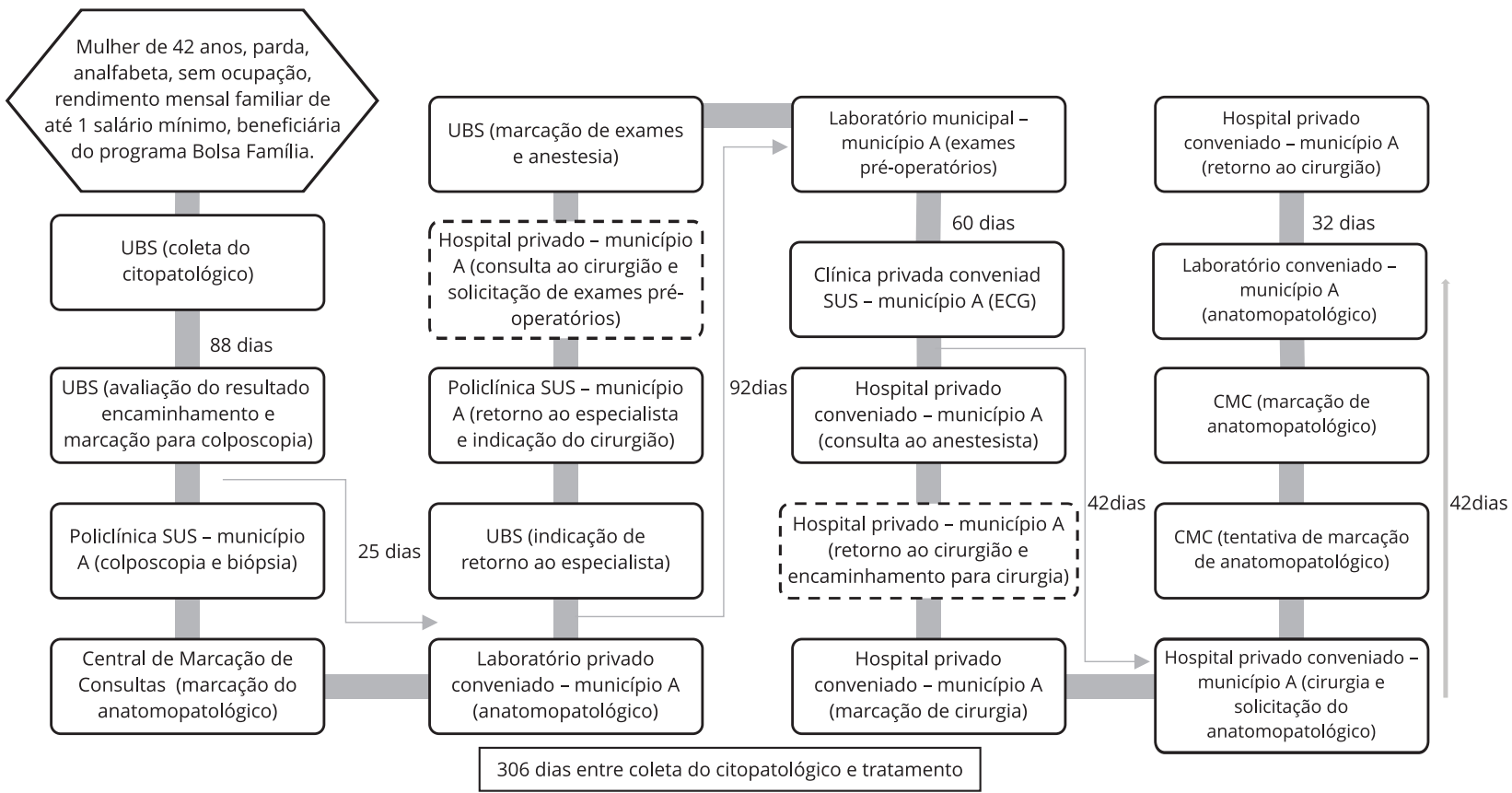

2b) Usuária do município C - município de menor porte.
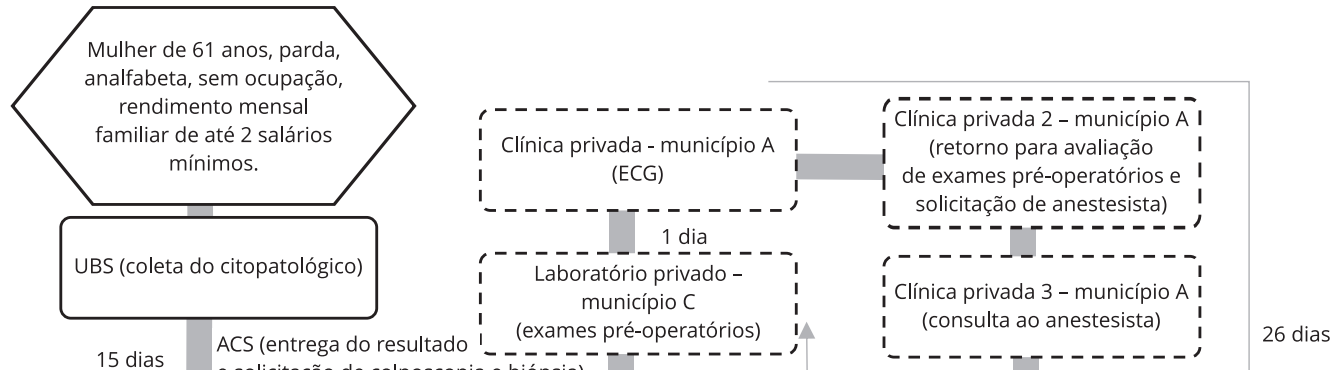

Ambulatório de especialidades -
município C (colposcopia, biópsia
e solicitação do anatomopatológico)

ia e biópsia)
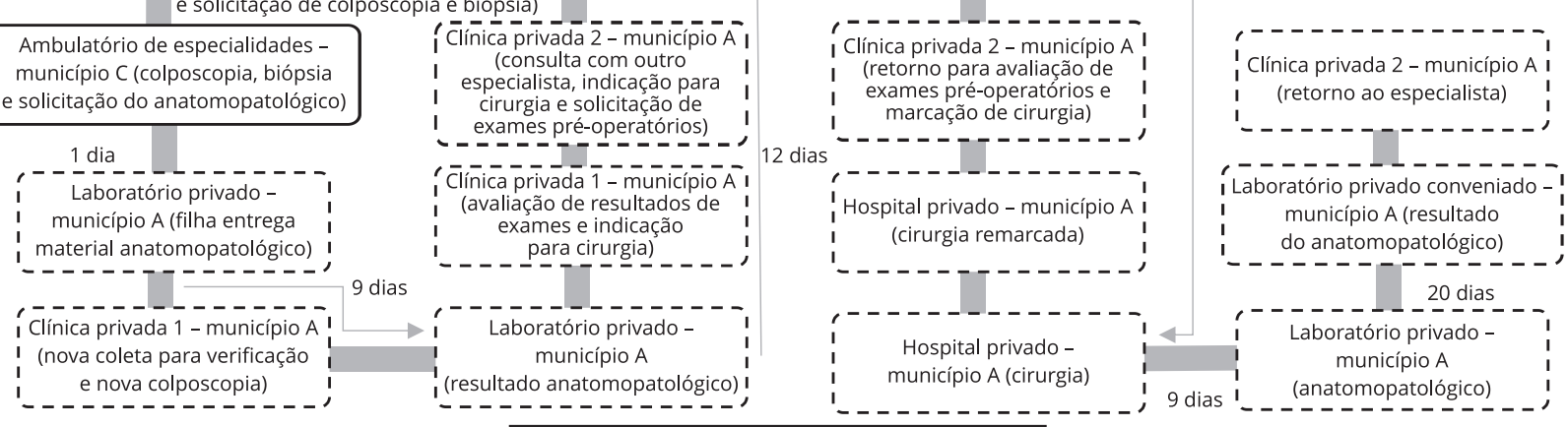

67 dias entre coleta do citopatológico e tratamento

serviço privado

serviço público ou privado conveniado

ECG: eletrocardiograma; SUS: Sistema Único de Saúde; UBS: unidade básica de saúde; USF: unidade de saúde da família. 
mulheres do município B, não foi possível eleger uma trajetória representativa. Para estimar os tempos de espera foram usados dados dos prontuários e resultados de exames.

Apesar dos problemas elencados, as usuárias, em geral, consideraram-se satisfeitas com o atendimento na rede de referência, com destaque ao cuidado ofertado por um ginecologista oncológico do município sede, por fornecer orientações e esclarecimento de dúvidas. Ademais, avaliaram positivamente a assistência prestada pela equipe dos hospitais do município sede. Também foram relatadas queixas, em serviços públicos e privados, relacionadas à falta de informações sobre o problema de saúde e procedimentos, além dos fluxos a serem percorridos na RAS. Algumas mulheres não tinham conhecimento sobre o tipo de procedimento cirúrgico ao qual foram submetidas. Atrasos no atendimento médico provocavam dificuldades de retorno aos povoados para as mulheres da zona rural. Todas essas situações geraram preocupação, receio de agravamento do problema e grande sofrimento, possivelmente evitáveis, como expresso em diversas falas.

\section{Sistemas de apoio e logístico: os "caminhos que eu mesma faço"}

Ao recorrerem ao pagamento de consultas e exames especializados pela dificuldade de acesso ou para agilizar o tratamento, as usuárias contaram com recursos de familiares, político, amigos e empréstimo bancário. Além do auxílio financeiro, o apoio da família foi muito presente na maioria das trajetórias assistenciais para a indicação de profissionais, marcação de exames/consultas e para o acompanhamento das mulheres. Também foi identificado apoio de instituição de caridade (casa de apoio a pessoas com câncer) no município sede.

Nenhuma das entrevistadas dos municípios do interior que necessitou deslocar-se à sede da região referiu garantia de transporte pela SMS, tampouco usuárias da zona rural do município A, cuja zona urbana, em alguns casos, fica a 100 quilômetros e onde não havia transporte público regular. Todas arcaram com os custos do transporte, muitas vezes, inadequado ao seu estado de saúde, além de aguardarem por muitas horas para o retorno à residência. Também foram recorrentes os deslocamentos apenas para agendamentos de consultas, exames e busca de resultados.

\section{Discussão}

As trajetórias assistenciais mostraram-se potentes para análise da rede regionalizada com base na experiência concreta de uso das mesmas. A utilização de uma condição traçadora amplificou o olhar sobre os pontos de interdição e passagem na busca por cuidados de usuárias que caminham pelo sistema de saúde, desde a APS até a atenção hospitalar. Ressalta-se não se tratar da análise de uma linha de cuidado, uma vez que as RAS devem se organizar de forma a garantir acesso a serviços de diferentes densidades tecnológicas, mas de uma ferramenta analítica para apreensão fina, precisa e compreensiva da experiência de mulheres no sistema de saúde.

De forma geral, os resultados indicaram maiores dificuldades de acesso à integralidade do cuidado nos municípios do interior da região de saúde, principalmente para as residentes naquele com menor disponibilidade de serviços e na zona rural da sede, indicando arranjos regionais pouco consolidados e uma porta de entrada para o sistema de saúde "entreaberta".

As usuárias reconhecem as equipes de APS como fonte regular de cuidado. Contudo, barreiras existem desde a entrada no sistema e, aqui, reforça-se a resiliência de uma certa organização do processo de trabalho que insiste em produzir o não cuidado por meio de filas, distribuição de senhas, entre outras. Assim, barreiras organizacionais somam-se a barreiras financeiras, especialmente em territórios rurais que exigem gastos com transporte.

De certo que zonas rurais, dispersas e com baixa densidade demográfica, demandam estratégias diferenciadas, com algum grau de inovação e viabilidade para a garantia de atenção integral e integrada, ainda por ser construída 20. Contudo, a premissa da universalidade e do direito à saúde não pode ser relativizada ou dirimida em função do local de residência, somando e ampliando os padrões de desigualdade econômica e social, prevalentes nos territórios rurais e que condicionam as piores condições de vida 21 . 
Como indicam outros estudos, a percepção de resolutividade da APS é condicionada pelas dificuldades de acesso aos níveis de maior densidade tecnológica 22,23, mas também por problemas intrínsecos a ela como abastecimento irregular de medicamentos e rotatividade de médicos. O relato da baixa frequência e irregularidade de atividades educativas e de convocação para o citopatológico de colo uterino parece indicar processos de trabalho pouco sinérgicos a uma concepção mais abrangente de APS, com atuação comunitária.

As trajetórias assistenciais tensionam a lógica formal de funcionamento dos serviços de saúde 5 . É preciso ressaltar que para todas as usuárias a referência à atenção especializada foi realizada pela APS e, para a maioria, o agendamento realizado pela UBS/USF, sinais de um papel de gatekeeper que vem se consolidando na região estudada 24 e no país 25 . No entanto, apenas a referência parece não ser suficiente para a garantia de um percurso mais seguro. A ausência de uma consulta pós-diagnóstico com a equipe de APS, mais notável entre as mulheres da zona rural e no município de menor porte, resultou em sofrimento possivelmente evitável, requerendo a compreensão de que o usuário deve fazer parte de seu processo de coordenação do cuidado 18,26.

A falta de acesso à informação também representa mitigação do direito à saúde. Neste estudo, nos casos em que a comunicação foi potente, esta representou uma ferramenta importante na produção do cuidado e um dos principais fatores de satisfação das usuárias 27 . Estudos convergem ao apontar o valor da comunicação horizontal entre profissional e usuário 11,28, o que contribui para a adesão ao tratamento.

$\mathrm{Na}$ transição para a atenção especializada, está o primeiro ponto de ruptura de alguma possível integração assistencial e de manutenção de desigualdades no acesso à saúde. Idas e vindas na busca por atenção especializada, nomeadas aqui "peregrinação", é observada em estudos que reconstroem trajetórias assistenciais em diferentes contextos 13,29. Achados mais positivos são registrados em municípios com melhor capacidade instalada 30 . Nesse ponto de transição, as equipes de APS desaparecem das trajetórias e inicia-se um caminhar solitário, muitas vezes, sem informações sobre a própria condição de saúde, com informações imprecisas sobre os fluxos assistenciais e sem apoio no serviço que deveria ser sua referência. Vale ressaltar que, cada vez mais, o papel de uma APS forte e robusta é reconhecido internacionalmente no cuidado de usuários com câncer, para prevenção, diagnóstico, seguimento e, quando necessário, cuidados paliativos 31 .

Maiores tempos de espera foram observados para as consultas com especialistas para as mulheres da área rural da sede se comparadas às munícipes da zona urbana, fortalecendo o conjunto de achados que ratificam desigualdades de acesso em função do município de residência 32 e as fragilidades na organização da rede regionalizada 33.

A composição de serviços públicos e privados por meio de desembolso direto está presente no caminhar da atenção especializada até a hospitalar, agravando os gastos catastróficos em saúde que, aqui, somam-se aos recursos dispendidos com transporte, maiormente no meio rural. O mix público-privado na composição da cesta de serviços é uma evidência presente em várias investigações $5,13,29,34$. Todavia, neste estudo, constatou-se a predominância de utilização de serviços públicos por mulheres do município sede e dos privados por aquelas do interior da região de saúde.

Os serviços privados foram utilizados no município sede da região mesmo quando não foram identificadas barreiras de acesso e oferta de serviços públicos. A expectativa do não acesso ou dos elevados tempos de espera condicionaram a conduta profissional: "agilizar" por meio do pagamento de "taxas" parecia ser a palavra de ordem, mesmo em casos com tempos de espera tecnicamente aceitáveis, resultado ratificado por outros estudos 34 . Silva et al. 11 denominaram "SUS Plus" o sistema no qual usuárias com CCU pagavam pela primeira consulta com especialistas, que também trabalhavam na rede pública, para facilitar o acesso ao SUS.

Tom ainda mais perverso assume o mix público-privado, com predomínio do privado, para os procedimentos pré-operatórios. Os resultados sinalizaram problemas que parecem não circunscritos apenas à deficiente oferta, mas a práticas clientelistas e privatização do espaço público ${ }^{35}$. Nesse sentido, a interferência de um profissional da APS, no exercício de um certo advocacy a favor da usuária, restabeleceu o fluxo pela rede pública. Tal achado reforça a necessidade da coordenação do cuidado, potencialmente capaz de proteger o usuário na relação assimétrica entre profissional/paciente e permitir a gestão clínica segura 36 . 
Frente aos obstáculos de acesso à atenção especializada, as usuárias buscaram apoio de conhecidos e políticos, que funcionaram como facilitadores, achado presente em outros estudos 11,13,29. Familiares como pontos de apoio legítimos assumiram a construção dos fluxos e condução das TA, evidência que reforça um conjunto de outras investigações 5,13,23.

Embora o início da caminhada tenha sido mediado pela APS, a continuidade foi garantida pela própria mulher, condutora de suas guias de referência. Esse é um ponto controverso na regulação assistencial. Qual o melhor caminho: voltar à APS para novos agendamentos ou referências realizadas do ponto atual de contato com o sistema? Não há uma resposta simples, contudo, delegar à usuária a responsabilidade pelos agendamentos no serviço especializado ou na central de marcação de consultas parece inadequado, sobretudo para residentes em áreas rurais ou que exigem gastos com transporte.

Para as usuárias que utilizaram a rede hospitalar, assim como na transição APS-atenção especializada, foi praticamente inexistente a menção a instrumentos de continuidade informacional. Achados similares confirmam estudos nacionais $3,5,11,13$ e internacionais 6 , ratificando a persistente e preocupante ausência de comunicação interprofissional.

A cirurgia no SUS foi garantida para as usuárias da sede da região, enquanto as de outros municípios realizaram desembolso direto. Observou-se também dificuldades para a realização do anatomopatológico. O acompanhamento pós-cirúrgico, igualmente, apresentou barreiras e, ao fim e ao cabo, as mulheres retornavam à APS para seguimento, na completa ausência de registro clínico, corroborando estudos que apontam a incipiência na comunicação entre níveis de atenção 37.

Este estudo soma-se ao conjunto de evidências que ratificam a fragilidade do desenho das redes regionalizadas, com manutenção de desigualdades, guardadas todas as limitações de um estudo de caso realizado em contexto específico. A opção pela pesquisa qualitativa pode não ser suficiente para a compreensão da organização e do acesso numa rede regionalizada, requerendo, assim, outras abordagens para ampliar o escopo dos resultados e possibilitar a triangulação de métodos. Na mesma direção, inquéritos ou mesmo entrevistas envolvendo diferentes interlocutores poderiam trazer à tona informações acerca dos encaminhamentos e tempos de espera, bem como, as condições estruturais e insumos disponíveis em estabelecimentos de saúde no conjunto dos municípios, dentre outros aspectos relevantes. Por sua vez, traz como potencialidade achados que revelam diferentes oportunidades de acesso entre municípios e intramunicípios, com clara desvantagem para usuárias do meio rural, mesmo que moradoras da sede da região de saúde.

Por fim, mais do que destacar as fragilidades do acesso e da organização das redes regionalizadas, a voz das usuárias sinaliza caminhos a seguir para a substituição de arranjos informais e privados que acabam por assumir a direção do sistema. Para além da necessária ampliação das bases materiais e financeiras capazes de sustentar uma APS abrangente, estratégias de coordenação horizontal no território, comunicação formal/informal entre profissionais de saúde e, especialmente, inclusão do usuário, de forma ativa, na definição de seu plano terapêutico revelaram-se estratégias potentes e possíveis para melhor a coordenação do cuidado e para a garantia de cuidado integral. 


\section{Colaboradores}

J. R. Galvão foi responsável pela realização do trabalho de campo, concepção, interpretação dos dados e redação do manuscrito. P. F. Almeida foi responsável pela concepção, interpretação dos dados, redação do manuscrito e aprovação da versão final. A. M. Santos e A. Bousquat participaram da interpretação dos dados, redação do manuscrito e aprovação da versão final.

\section{Informações adicionais}

ORCID: Jôse Ribas Galvão (0000-0003-4131-1393); Patty Fidelis de Almeida (0000-0003-1676-3574); Adriano Maia dos Santos (0000-0001-9718-1562); Aylene Bousquat (0000-0003-2701-1570)

\section{Agradecimentos}

O presente artigo faz parte do estudo: Coordenação do cuidado pela Atenção Primária à Saúde em Redes Regionalizadas: Contrastando Experiências Sul-Americanas, financiado por meio de Bolsa do Programa de Pós-Doutorado Júnior do CNPq (Processo 150036/2117-5), concedida à segunda autora, e de seu subprojeto: Avaliação da Coordenação do Cuidado em Regiões de Saúde: Estudo a Partir de uma Situação Marcadora.

\section{Referências}

1. European Commission. Tools and methodologies to assess integrated care in Europe. Luxembourg: Publications Office of the European Union; 2017.

2. Viana ALA, Bousquat A, Melo GA, Negri Filho A, Medina MG. Regionalização e redes de saúde. Ciênc Saúde Colet 2018; 23:1791-8.

3. Santos L. Região de saúde e suas redes de atenção: modelo organizativo-sistêmico do SUS. Ciênc Saúde Colet 2017; 22:1281-9.

4. Almeida PF, Santos AM. Atenção Primária à Saúde: coordenadora do cuidado em redes regionalizadas? Rev Saúde Pública 2016; 50:80.

5. Bousquat A, Giovanella L, Campos EMS, Almeida PF, Martins CL, Mota PHS, et al. Atenção primária à saúde e coordenação do cuidado nas regiões de saúde: perspectiva de gestores e usuários. Ciênc Saúde Colet 2017; 22:1141-54.

6. Vargas I, Garcia-Subirats I, Mogollón-Pérez AS, Paepe P, Silva MRF, Unger JP, et al. Patient perceptions of continuity of health care and associated factors. Cross-sectional study in municipalities of central Colombia and north-eastern Brazil. Health Policy Plan 2017; 32:549-62.

7. Martin LT, Plough A, Carman KG, Leviton L, Bogdan O, Miller CE. Strengthening integration of health services and Systems. Health Aff (Millwood) 2016; 35:1976-81.

8. Dzuba IG, Calderon R, Bliesner S, Luciani S, Amado F, Jacob M. A participatory assessment to identify strategies for improved cervical cancer prevention and treatment in Bolivia. Rev Panam Salud Pública 2005; 18:53-63.

9. Wiesner C, Cendales R, Murillo R, Piñeros M, Tovar S. Seguimiento de mujeres con anormalidad citológica de cuello uterino, en Colombia. Rev Salud Pública 2010; 12:1-13.

10. Costa RFA, Longatto-Filho A, Lima Vazquez F, Pinheiro C, Zeferino LC, Fregnani JHTG. Trend analysis of the quality indicators for the Brazilian cervical cancer screening programme by region and state from 2006 to 2013. BMC Cancer 2018; 18:126.

11. Silva MRF, Braga JPR, Moura JFP, Lima JTO. Continuidade Assistencial a mulheres com câncer de colo de útero em redes de atenção à saúde: estudo de caso, Pernambuco. Saúde Debate 2016; 40:107-19.

12. Silveira R, Feitosa A, Lofego J, Souza LC. Trajetórias assistenciais e itinerários terapêuticos como práticas avaliativas da integralidade em Rio Branco. In: Pinheiro R, Silva Jr. AG, Mattos RA, organizadores. Atenção básica e integralidade: contribuições para estudos de práticas avaliativas em saúde. Rio de Janeiro: Centro de Estudos e Pesquisa em Saúde Coletiva, Instituto de Medicina Social, Universidade do Estado do Rio de Janeiro/Abrasco; 2011. p. 215-24. 
13. Fausto MCR, Campos EMS, Almeida PF, Medina MG, Giovanella L, Bousquat A, et al. Itinerários terapêuticos de pacientes com acidente vascular encefálico: fragmentação do cuidado em uma rede regionalizada de saúde. Rev Bras Saúde Matern Infant 2017; 17 Suppl 1:563-73.

14. Gerhardt TE, Burrille A, Müller TL. Estado da arte da produção científica sobre itinerários terapêuticos no contexto brasileiro. In: Gerhardt TE, Pinheiro R, Ruiz ENF, Silva Junior AG, organizadores. Itinerários terapêuticos: integralidade no cuidado, avaliação e formação em saúde. Rio de Janeiro: Centro de Estudos e Pesquisa em Saúde Coletiva, Instituto de Medicina Social, Universidade do Estado do Rio de Janeiro/Abrasco; 2016. p. 27-97.

15. Kleinman A. The illness narratives: suffering, healing, and the human condition. New York: Basic Books; 1988.

16. Bahia. GeoPortal de informações em saúde. http://geolivre.saude.ba.gov.br/geoportal/ map.phtml?language $=$ br (acessado em 04/Jan/ 2019).

17. Almeida PF, Fausto MCR, Giovanella L. Fortalecimento da atenção primária à saúde: estratégia para potencializar a coordenação dos cuidados. Rev Panam Salud Pública 2011; 29:84-95.

18. McDonald KM, Schultz E, Albin L, Pineda N, Lonhart J, Sundaram V, et al. Care coordination atlas version 4. Rockville: Agency for Healthcare Research and Quality; 2014.

19. Minayo MCS. O desafio do conhecimento: pesquisa qualitativa em saúde. 14a Ed. São Paulo: Editora Hucitec; 2012.

20. Richard L, Furler J, Densley K, Haggerty J, Russel G, Levesque J-F, et al. Equity of access to primary healthcare for vulnerable populations: the impact international online survey of innovations. Int J Equity Health 2016; 15:120.

21. Coimbra Jr. CEA. Saúde rural no Brasil: tema antigo mais que atual. Rev Saúde Pública 2018; 52 Suppl 1:2s.

22. Cecílio LCO, Reis AAC. Apontamentos sobre os desafios (ainda) atuais da atenção básica à saúde. Cad Saúde Pública 2018; 34:e00056917.

23. Xavier RB, Bonan C, Silva KS, Nakano AR. Itinerários de cuidados à saúde de mulheres com história de síndromes hipertensivas na gestação. Interface (Botucatu) 2015; 19:1109-20.

24. Santos AM. Redes regionalizadas de atenção à saúde: desafios à integração e à coordenação do cuidado. Salvador: Edufba; 2018.

25. Fausto MCR, Giovanella L, Mendonça MHM, Seidl H, Gagno J. A posição da Estratégia Saúde da Família na rede de atenção à saúde na perspectiva das equipes e usuários participantes do PMAQ-AB. Saúde Debate 2014; 38:1333.

26. Almeida PF. Atenção primária à saúde no Brasil e os 40 anos de Alma-Ata: reconhecer os desafios para seguir adiante. Cad Saúde Pública 2018; 34:e0136118.
27. Brito-Silva K. Avaliação da integralidade no cuidado ao câncer do colo uterino: uso da condição marcadora em um estudo misto [Tese de Doutorado]. São Paulo: Faculdade de Saúde Pública, Universidade de São Paulo; 2013.

28. Jesus RPFS, Espírito Santo ACG, Mendes MFM, Samico IC. Percepção dos profissionais sobre a coordenação entre níveis de atenção à saúde em dois municípios pernambucanos de grande porte. Interface (Botucatu) 2018; 22:423-34.

29. Lofego J, Pinheiro R. Itinerário terapêutico no controle do câncer de colo uterino: uma análise sob a perspectiva da integralidade em saúde e do direito à comunicação. In: Gerhardt TE, Pinheiro R, Ruiz ENF, Silva Junior AG, organizadores. Itinerários terapêuticos: integralidade no cuidado, avaliação e formação em saúde. Rio de Janeiro: Centro de Estudos e Pesquisa em Saúde Coletiva, Instituto de Medicina Social, Universidade do Estado do Rio de Janeiro/Abrasco; 2016. p. 306-26.

30. Brito-Silva K, Bezerra AFB, Chaves LDP, Tanaka OY. Integralidade no cuidado ao câncer do colo do útero: avaliação do acesso. Rev Saúde Pública 2014; 48:240-8.

31. Rubin G, Berendsen A, Crawford SM, Dommett R, Earle C, Emery J, et al. The expanding role of primary care in cancer control. Lancet Oncol 2015; 16:1231-72.

32. Arruda NM, Maia AG, Alves LC. Desigualdade no acesso à saúde entre as áreas urbanas e rurais do Brasil: uma decomposição de fatores entre 1998 e 2008. Cad Saúde Pública 2018; 34:e00213816.

33. Alves MO, Magalhães SCM, Coelho BA. A regionalização da saúde e a assistência aos usuários com câncer de mama. Saúde Soc 2017; 26:141-54.

34. Raupp LM, Dhein G, Medeiros CRG, Grave MTQ, Saldanha OMFL, Santos MV, et al. Doenças crônicas e trajetórias assistenciais: avaliação do sistema de saúde de pequenos municípios. Physis (Rio J.) 2015; 25:615-34.

35. Silva JF, Carvalho BG, Domingos CM. A governança e a relação público-privado no cotidiano das práticas em municípios de pequeno porte. Ciênc Saúde Coletiva 2018; 23:3179-88.

36. Aller MB, Vargas I, Coderch J, Calero S, Cots F, Abizanda M, et al. Development and testing of indicators to measure coordination of clinical information and management across levels of care. BMC Health Serv Res 2015; 15:323.

37. Lima NC, Baptista TWF, Vargas EP. Ensaio sobre 'cegueiras': itinerário terapêutico e barreiras de acesso em assistência oftalmológica. Interface (Botucatu) 2017; 21:615-27. 
Abstract

The article aims to assess organization and access to the Healthcare Network in a health region, from the perspective of female users. Healthcare trajectories were constructed for women with highgrade cervical squamous intraepithelial lesions, covered by different modalities of primary health care (PHC) in urban and rural areas, in municipalities in the interior, and in the capital city of the Northeast health region, Brazil. Women used PHC as their regular service for prevention and care, but reported barriers to access to medical appointments, especially in rural areas. Positive assessments were associated with positive reception by the health service and representation of the basic health unit or family health unit as the available locus for care. A perception of low case-resolution capacity in PHC was associated with delays in scheduling referrals, irregular/insufficient supply of medicines, and physician turnover. Women reported difficulty in access to specialized care, especially in municipalities in the interior, with the use of public and private services. All the users in the capital city of the health region underwent confirmatory diagnostic tests and surgeries in the Brazilian Unified National Health System (SUS). Support from family members, friends, and local politicians were part of the healthcare trajectories. Interprofessional communication was nearly nonexistent and health professional-patient communication was precarious. The regionalized network appeared disconnected and with confusing flows in terms of guaranteeing timely access for users in municipalities in the interior and with additional difficulties for those in rural areas, even in the capital city, revealing incompleteness in regional arrangements and persistence of inter-and intramunicipal inequalities in access.

Comprehensive Health Care; Systems Integration; Regional Health Planning; Uterine Cervical Neoplasms

\section{Resumen}

El artículo tiene como objetivo evaluar la organización y el acceso a la Red de Atención de Salud en una región de salud, desde la perspectiva de las usuarias. Se construyeron trayectorias asistenciales de mujeres con una lesión intraepitelial escamosa de alto grado en el cuello del útero, adscritas a diferentes modalidades de atención primaria de salud (APS) de zonas urbanas y rurales, en municipios del interior y de la sede de región de salud del Nordeste, Brasil. Las mujeres utilizaban la APS, como servicio de búsqueda regular para acciones preventivas y asistenciales, pero informaron sobre barreras de acceso para consultas médicas, sobre todo en las zonas rurales. Las evaluaciones positivas se vincularon con la acogida y la representación de la unidad básica de salud/ unidad de salud familiar (UBS/USF) como un lugar disponible para cuidados. La percepción de baja resolutividad de la APS estuvo vinculada a la tardanza para las citas con el especialista, suministro irregular/insuficiente de medicamentos y rotatividad de médicos. Las mujeres indicaron dificultades de acceso a la atención especializada, aún más evidentes en los municipios del interior de la región, con utilización de servicios públicos y privados. Todas las usuarias de la sede de la región realizaron exámenes de confirmación diagnóstica y cirugía a través del Sistema Unico de la Salud. El apoyo de familiares, amigos y politicos se cruzó con las trayectorias asistenciales. La comunicación interprofesional fue casi inexistente y entre profesional-usuaria precaria. La red regionalizada se presentó desarticulada y con flujos desordenados, no garantizando el acceso oportuno a las usuarias de los municipios del interior, y presentando dificultades adicionales para las procedentes del área rural, incluso en el municipio sede, desvelando lo incompleto de los acuerdos regionales y la existencia de desigualdades de acceso inter-e intramunicipal.

Atención Integral de Salud; Integración de Sistemas; Regionalización; Neoplasias del Cuello Uterino
Recebido em 08/Jan/2019

Versão final reapresentada em 18/Abr/2019

Aprovado em 07/Jun/2019 